\title{
Development of A Fixed-Wing mini UAV with Transitioning Flight Capability
}

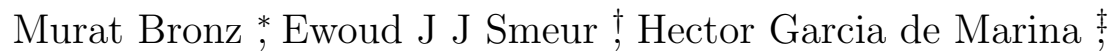 \\ and Gautier Hattenberger $\S$ \\ ENAC, F-31055 Toulouse, France \\ University of Toulouse, F-31400 Toulouse, France
}

\begin{abstract}
This study presents the development of the transitioning vehicle Cyclone, which has been specifically designed for meteorological and agricultural applications. The mission requirements demand take-off and landing from a small area and the ability to cope with high wind speeds. In contrast with recent suggestions, our proposed design aims to be closer to a fixed-wing airplane rather than a rotary wing. In particular, the design focuses on a tiltbody style transitioning vehicle with blown-wing concept. The propeller wing interaction is calculated using a semi-empirical method. The total wing span and wing surface area are decided according to the mission performance requirements. For the control of the vehicle, incremental nonlinear dynamic inversion is used. This control method does not need the modeling of external forces or moments and is able to counteract the strong aerodynamic forces and moments acting on the vehicle through the feedback of its angular acceleration. Together with the design phases and manufacturing process, several test flights are presented. Particular difficulties of the proposed design are discussed, including lack of providing sufficient pitch-up moment and control reversal during descent. The test flights demonstrate the vertical take-off and landing capabilities of the vehicle, as well as its transitioning into forward flight from hovering, and vice versa, for an efficient mission performance.
\end{abstract}

\section{Introduction}

Operational efficiency and compactness of small Unmanned Air Vehicles (UAV) increased their use in several areas such as atmospheric research and agricultural applications. ${ }^{1}$ These missions usually demand for takeoff and landing from a small area, where rotary-wing configurations are more suitable for their vertical or short take-off and landing capabilities. However, good performance is expected on endurance, range and also on climbing and high-speed flight, which are obtained more efficiently in fixed-wing configurations.

This complex mission profile has already been addressed by the unique flight characteristics of Vertical/Short Takeoff and Landing (V/STOL) aircraft. However, their additional complexity reduces the operational efficiency, which is the focus point for small UAV in the first place. This problem can be improved with a mission oriented optimization of the vehicle as previously shown by Bronz and Hattenberger. ${ }^{2,3}$ This approach requires a clear picture about how the final performance of the vehicle is affected by the main design variables.

Figure 1 shows some of the recent V/STOL trends. The first image from left shows ISAE's tilt-body $M A V I O N,{ }^{4}$ which is a good example of a hand-release vertical take-off and afterwards transition to cruise flight concept. The tiltwing demonstrator form RWTH Aachen University, ${ }^{5}$ shown second, is capable of vertical take-off by tilting its wing and then transition for an efficient cruise. The DelftaCopter ${ }^{6}$ shown third, uses a helicopter setup, with a single rotor and a swash plate, to control the attitude. The collective pitch enables optimization of the propeller pitch angle for both forward flight and hover. The GL-10, shown last, is a good example of a highly-Distributed Electric Propulsion (DEP) system, which can adapt

*Assistant Professor on Applied Aerodynamics, ENAC UAV Lab, F-31055 Toulouse, France

${ }^{\dagger}$ Ph.D. Candidate, MAVLab TUDelft, 2629 HS Delft, The Netherlands

${ }^{\ddagger}$ Researcher on Guidance, Navigation, and Control, ENAC UAV Lab, F-31055 Toulouse, France

$\S$ Assistant Professor on Flight Dynamics, ENAC UAV Lab, F-31055 Toulouse, France 

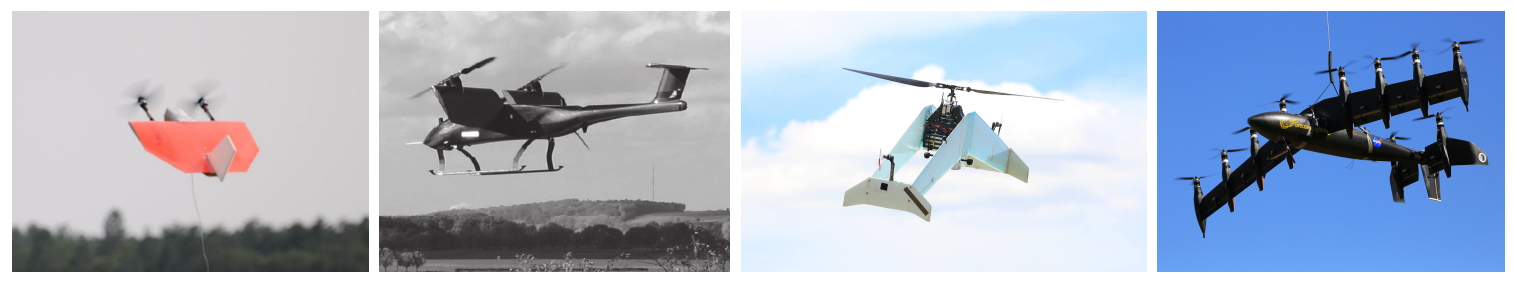

Figure 1. Tilt-Body micro air vehicle MAVION from ISAE, a tiltwing demonstrator from RWTH Aachen University, the DelftaCopter from MAVLab TUDelft, and a small demonstrator of NASA's $G L-10$.

the rotation rate of its individual propellers located on the leading edge of the wing, in order to increase efficiency. On DEP systems, the wings are immersed inside the distributed propeller slipstream, which increases the dynamic pressure over them. Consequently, this results in a significant wing surface reduction for traditional aircraft. This makes them more efficient during the cruise flight conditions as the drag caused by the wing surface that is only needed for the take-off and landing is reduced.

\section{A. Present Work}

This work focuses on the design and flight testing of a fixed-wing aircraft with take-off and landing capabilities, including full equilibrium low-speed flight during the transition phase. The design of such a configuration requires a good understanding of the interaction between the DEP system and the airframe. Unfortunately, the propeller wing interaction is very complex to model without certain simplifications, especially with high-fidelity methods, as was concluded by Veldhuis. ${ }^{8}$

The paper is organized as follows. In Section II, the basis of the aerodynamic calculations are introduced based on Jameson, ${ }^{9}$ who has developed semi-empirical formulations in order to estimate the force and moment generation on V/STOL vehicles. Section III describes the design philosophy and the steps to select the main design variables such as wing span, wing surface area, and battery weight in order to obtain optimum performance for a given mission definition. In Section IV the whole manufacturing process of the prototype vehicle is shown. Section V explains the control system applied to the vehicle based on incremental nonlinear dynamic inversion. Finally, in Section VI, flight tests demonstrating the vertical, horizontal, and transitioning phases of the vehicle are shown.

\section{Aerodynamic Model for Propeller Wing Combination}

During the development of the vehicle, the complex propeller and wing interaction is modeled by a semiempirical method from Jameson. ${ }^{9}$ Additional viscous drag, moment, and equilibrium case calculations are added by Bronz and Drouin. ${ }^{10}$ The method has been already explained completely with detail in those references, ${ }^{9,10}$ however in order to keep the consistency of this paper, the main points will be repeated in the following subsections.

An arbitrary number of propeller slipstreams are defined, with individual thrust, actuator disk area, position and orientation. The method is mainly based on momentum theory, which means that the swirl effects of the propeller slipstream are not modeled.

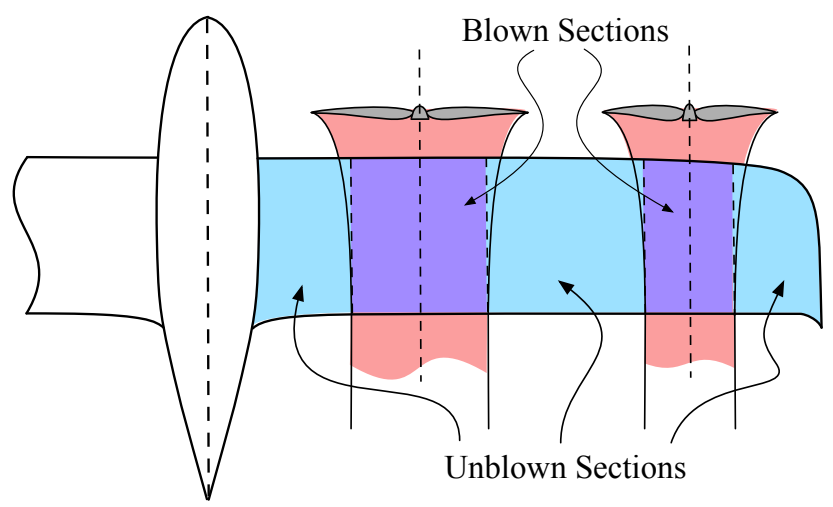

Figure 2. The creation of wing sections according to the fully developed propeller slipstream width. Note that for each thrust value the sections dynamically are changed in order to take the contraction into account. 


\section{A. Propeller Forces}

The propeller forces are modeled according to actuator disk theory. For a given propeller thrust $T$ and actuator area $S_{p}$, the ratio $\mu$ between the free stream $V_{\infty}$ and propeller jet slipstream $V_{j}$, is given by

$$
\mu=\frac{V_{\infty}}{V_{j}}=\sqrt{1-\frac{T}{0.5 \rho V_{j}^{2} S_{p}}}
$$

It is assumed that each individual slipstream keeps a circular cross section such that their contraction is estimated with

$$
b_{p c}=b_{p} \sqrt{\frac{1+\mu}{2}}
$$

where $b_{p}$ is the propeller disk diameter, and $b_{p c}$ is the fully developed contracted slipstream diameter. Once the contracted slipstream diameter is calculated, the wing can be separated into the sections that are inside and outside of the propeller slipstream as shown in Figure 2. Note that the fully developed propeller slipstream is taken for simplification reasons.

Inclined propellers deflect the free stream, consequently changing the angle of attack of the wing inside this slipstream. This downwash $\epsilon$ can be determined using the inflow angle of the propeller $\alpha_{j}$ as is described by Ribner ${ }^{11}$ and De Young. ${ }^{12}$

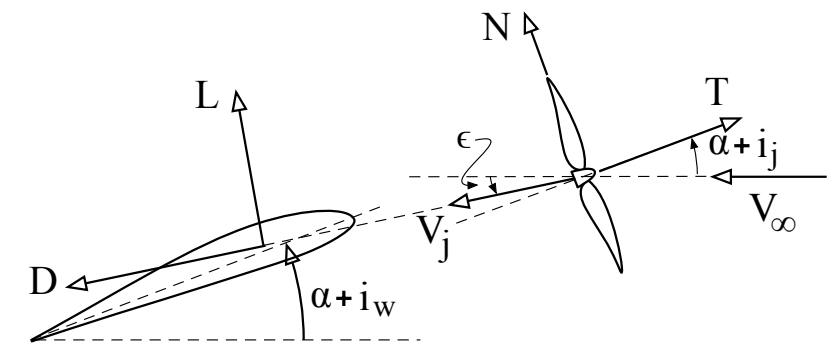

Figure 3. The wing incidence angle $i_{w}$, jet incidence angle $i_{j}$, angle of attack of the fuselage $\alpha$ with the resultant lift $L$, drag $D$, thrust $T$ and the propeller normal force $N$, propeller downwash $\epsilon$, jet slipstream $V_{j}$.

\section{B. Actuator Inflow Angle Change due to Wing, Fuselage and Other Propeller Jets Upwash}

Each actuator is influenced by the fuselage, wing, and the other propeller jets. Taking these effects into account, the inflow angle for each jet is the sum of

$$
\alpha_{j}=\alpha+i_{j}+U_{w}\left(\alpha+i_{w}\right)+U_{f} \alpha+\sum_{\text {otherjets }} U_{o_{j}} \epsilon
$$

where $U_{w}$ is the upwash due to the wing, $U_{f}$ is the upwash due to the fuselage and $U_{0_{j}}$ is the upwash due to the other propeller slipstreams. For each propeller slipstream, $\alpha_{j}$ can be written as

$$
\begin{aligned}
\alpha_{j 1} & =\alpha+i_{j_{1}}+U_{w}\left(\alpha+i_{w}\right)+U_{f} \alpha+U_{o j_{12}} \epsilon_{2}+\cdots+U_{o j_{1 n}} \epsilon_{n} \\
\alpha_{j 2} & =\alpha+i_{j_{2}}+U_{w}\left(\alpha+i_{w}\right)+U_{f} \alpha+U_{o j_{21}} \epsilon_{1}+\cdots+U_{o j_{2 n}} \epsilon_{n} \\
\alpha_{j 3} & =\alpha+i_{j_{3}}+U_{w}\left(\alpha+i_{w}\right)+U_{f} \alpha+U_{o j_{31}} \epsilon_{1}+\cdots+U_{o j_{3 n}} \epsilon_{n} \\
& \vdots \\
\alpha_{j n} & =\cdots
\end{aligned}
$$

for a half wing with $n$ propellers mounted on the leading edge. The quantity $U_{o j_{12}}$ presents the upwash effect of the second actuator on the first one. As long as there is a fuselage between the propellers separating the slipstreams, the upwash effects coming from the other wing can be neglected, leading to a set of linear equations of the form $A x=b$. Therefore, the inflow angles $\alpha_{j}$ can be directly calculated. Similarly, wing 
inflow angles for sections that are inside the freestream and that are inside the propeller slipstream can be calculated as follows:

$$
\begin{aligned}
\alpha_{w_{j 1}} & =\alpha+i_{w}+U_{f} \alpha \\
\alpha_{w_{j \mu}} & =\alpha_{w_{j 1}}-\epsilon+\sum_{j e t s} U_{\infty_{j}} \epsilon
\end{aligned}
$$

The propeller normal force can be calculated with the assumption of being proportional to the sine of the inflow angle $\alpha_{j}$, i.e.,

$$
C_{N}=C_{N_{\alpha}} \sin \alpha_{j}
$$

The resulting lift slope in a slipstream is a function of the influenced mass flow around the wing. The wing in free stream is influenced by a mass flow that is passing through a circular surface $S_{\infty}$ with area $\pi b^{2} / 4$, which contains the wing tips. However, the wing in a slipstream, passing through the surface $S_{j}$, influences a smaller mass flow resulting in a reduction in the effective span or aspect ratio of the wing which is given by

$$
A R_{0}=\frac{A R}{1+p}
$$

where $p$ is a positive value determined according to the jet slipstream aspect ratio.

The resultant lift can be simply estimated by the superposition of forces over the wing in free stream and the individual parts that are immersed in slipstreams. The individual parts that are immersed in slipstreams are calculated as isolated planforms. Thus, the additional increase on each isolated planform is simply the difference between the planform in free stream $V_{\infty}$, and the planform immersed in a jet slipstream $V_{j}$ moving with a forward speed of $V_{\infty}$. The difference in lift is then described by

$$
\Delta L=\frac{1}{2} \rho S_{w_{j}}\left(V_{j}^{2} C L_{\alpha_{j \mu}} \alpha_{w_{j \mu}}-V_{\infty}^{2} C L_{\alpha_{\infty}} \alpha_{w_{j_{\infty}}}\right)
$$

where $C L_{\alpha_{j \mu}}$ is the lift slope of the wing part that is inside the jet slipstream with a velocity ratio of $\mu=V_{\infty} / V_{j}$ by taking the aspect ratio as $b_{j} / S_{w_{j}}$. The quantity $C L_{\alpha_{\infty}}$ is the lift slope of the same wing part in a freestream, or in other words when $\mu=1, \alpha_{w_{j \mu}}$ is the angle of attack of the wing in jet slipstream, and $\alpha_{w_{j}}$ is the angle of attack of the same part in free stream. It should be noted that the angle of attack of the wing portion inside the jet slipstream is reduced, compared to the angle of attack in the free stream, by the slipstream downwash $\epsilon$. Additionally, an inclined slipstream to the free stream generates an external upwash, which can be approximated by assuming the slipstream as a falling cylinder model. Then, the upwash at a distance $y$ from the center axis of the slipstream can be written as $\epsilon b_{j}^{2} / 2 / y^{2}$.

According to Jameson, ${ }^{9}$ the average upwash over the external part of the wing (outside the propeller jets) is approximately $\frac{S_{w_{j}}}{S} \epsilon$. Taking all upwash effects of propeller jets, the increase in lift can be calculated by multiplying the sum of all slipstream upwash angles with the unblown surface area and the lift slope of the complete wing in freestream $C L_{\alpha_{\infty}}$.

For small angles of attack, the lift force generated by the wing can be found by adding up the freestream lift of the complete wing, the additional lift created from the unblown parts of the wing because of the upwash effects of the jets, and finally the additional lift of the blown sections coming from the dynamic pressure increase because of the jet velocities. This can be encompassed with the following expression

$$
L=0.5 \rho V_{\infty}^{2} S C L_{\alpha_{\infty}}+0.5 \rho V_{\infty}^{2} S C L_{\alpha_{\infty}}\left(S-\sum_{j e t s} S_{w_{j}}\right) \sum_{j e t s} \frac{S_{w_{j}}}{S} \epsilon+\sum_{j e t s} \Delta L_{j}
$$

A similar approach can be used to calculate the additional induced drag for the wing sections that are inside the slipstream.

$$
\begin{gathered}
\Delta D_{i}=0.5 \rho V_{\infty}^{2} S_{w_{j}} C L_{j_{\infty}}\left(\alpha_{i_{j}}-\alpha_{i_{\infty}}\right)+\Delta L \alpha_{i_{j}} \\
D_{i}=0.5 \rho V_{\infty}^{2} S k C L_{\infty}^{2}+\sum_{j e t s} \Delta D_{i_{j}}
\end{gathered}
$$




\section{Effect of Flaps}

The effect of the flaps is modeled by an increase of the wing angle of attack as presented by Jameson. ${ }^{9}$ The effective wing incidence angle $i_{w_{\infty}}$ becomes

$$
i_{w_{\infty}}=i_{w}+\alpha / \delta_{\infty} \delta_{f}
$$

where $\delta_{f}$ is the flap deflection, and the flap effectiveness of the three dimensional wing in free stream $\alpha / \delta_{\infty}$ is given by

$$
\alpha / \delta_{\infty}=\frac{\sqrt{\alpha / \delta_{2 D}}+\alpha / \delta_{2 D} \frac{A R+4.5}{A R+2} A R}{\sqrt{\alpha / \delta_{2 D}}+\frac{A R+4.5}{A R+2} A R}
$$

Likewise for a wing section inside a propeller slipstream, the effective wing incidence $i_{w_{j \mu}}$ becomes

$$
i_{w_{j \mu}}=i_{w}+\alpha / \delta_{j \mu} \delta_{f}
$$

\section{Forces}

So far only longitudinal flight dynamics are considered, so the lateral force and moments are assumed to be zero $F_{y}=M_{x}=M_{z}=0$ as in equilibrium. The contribution of the wing lift $L_{\infty}$, drag $D_{\infty}$, propeller thrust $T$, and propeller normal force $N$ will be taken into account as

$$
\begin{aligned}
& F_{z}=L_{\infty}+\sum_{j e t s} T \sin \left(\alpha+i_{j}\right)+\sum_{j e t s} N \cos \left(\alpha+i_{j}\right) \\
& F_{x}=D_{\infty}-\sum_{j e t s} T \cos \left(\alpha+i_{j}\right)+\sum_{j e t s} N \sin \left(\alpha+i_{j}\right)
\end{aligned}
$$

\section{E. Moments}

The pitching moment is calculated by the resultant wing forces and moments. The thrust moment arm $d_{T_{j}}$ does not change during the pitch variation. Though variation of the pitch angle affects the position of the aerodynamic center of the wing, as was experimentally shown by Draper and Kuhn, ${ }^{13}$ in this analysis we will assume it to be fixed. As the aircraft is capable of increasing its pitch attitude to 90 degrees, the moment arm between the wing aerodynamic center and the center of gravity of the aircraft changes during this rotation as it is shown in Figure 4. Therefore, the lift force moment arm $d_{L}$ and drag

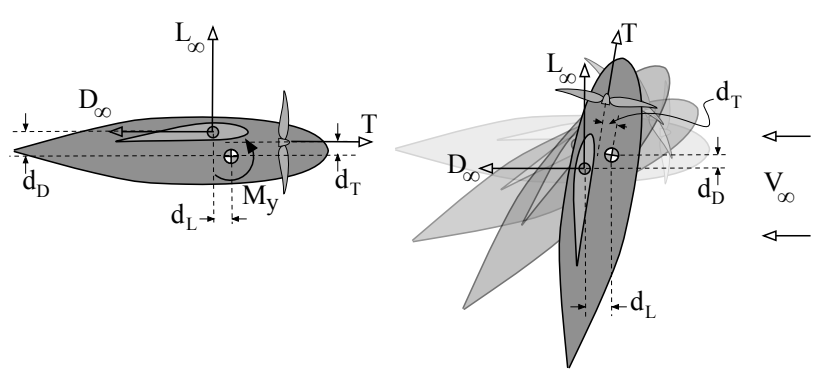

Figure 4. Illustration of the moment arm length variation during pitch attitude change. force moment arm $d_{D}$ have to be calculated at every angle of attack. Finally, the total pitching moment of the aircraft is calculated by

$$
M_{y}=M_{\text {wing }_{A C}}-\sum_{j e t s} T_{j} d_{T_{j}}-L_{\infty} d_{L}+D_{\infty} d_{D}
$$

\section{F. Equilibrium Calculations}

In order to fly in an equilibrium state at every flight speed $\left(V_{\infty}\right)$, the aircraft must satisfy

$$
F_{x}=0 \quad, \quad F_{z}=0 \quad, \quad M_{y}=0,
$$

via thrust $(\mathrm{T})$ and the elevator deflections $\left(\delta_{e}\right)$. For steady cases, this is obtained by finding the roots via the Newton-Raphson technique. This method requires an initial guess of the control inputs, close to the actual inputs necessary, in order to find the convergence. This initial guess can be easily calculated for the cruise flight case. The converged values of the cruise condition are then used as initial values for a slightly slower speed, and so forth. 


\section{Design of the prototype}

This section presents the main steps and decisions for the design of the prototype. The methods explained in the previous sections are embedded into a program that takes many input variables, such as wing-span, wing-surface, motor number, propeller size, location, and incidence angles, thrust of each propeller, etc. With the help of this program the lift, drag, and moment of any candidate configuration can be calculated for a given flight velocity, propeller thrust and control surface deflection.

\section{A. Design Evaluation}

The primary design driver is the operational efficiency of the vehicle, therefore the priority of the iterative optimization is to obtain the smallest and the most compact system that can satisfy the mission requirements. Table 1 shows the constraints for a representative mission.

\begin{tabular}{llcll}
\hline Max Dimension & $\leq$ & 1.0 & {$[\mathrm{~m}]$} & (Limiting the wing span) \\
Cruise Speed & $=$ & $14-16$ & {$[\mathrm{~m} / \mathrm{s}]$} & (Typical cruise speed) \\
Dash Speed & $\geq$ & 25 & {$[\mathrm{~m} / \mathrm{s}]$} & (Penetrating into wind) \\
Endurance & $\geq$ & 90 & {$[\mathrm{~min}]$} & (At cruise conditions) \\
Payload Mass & $=$ & 0.2 & {$[\mathrm{~kg}]$} & (Typical payload) \\
Total Mass & $\leq$ & 2.0 & {$[\mathrm{~kg}]$} & (Restricted by the regulations) \\
\hline
\end{tabular}

Table 1. Constraints of a representative mission.

A multi disciplinary optimization could have been applied to the development, with a detailed structural model and weight estimation models included. However, for the simplicity and rapid development of the prototype, the following highly simplified structural weight model is used

$$
W_{\text {structural }}=\mathcal{K} S_{\text {ref }} b
$$

where $\mathcal{K}$ is the structural weight coefficient which is found from previously build vehicles, $S_{r e f}$ is the reference wing surface area, and $b$ is the wing span.

Afterwards, the total weight is estimated by summing up all of the component weights

$$
W_{\text {total }}=W_{\text {structural }}+W_{\text {electronics }}+W_{\text {propulsion }}+W_{\text {battery }}+W_{\text {payload }}
$$

The weight of the on-board electronics is already minimized and does not depend on the aircraft size in this scale as we narrowed down the size limitation. Figure 5 shows a picture of the autopilot board called Chimera $v 1.0^{\text {a }}$, which weighs approximately 30 grams. It runs the Paparazzi open-source autopilot system, which contains software for communication, servo drivers, state estimation, etc.

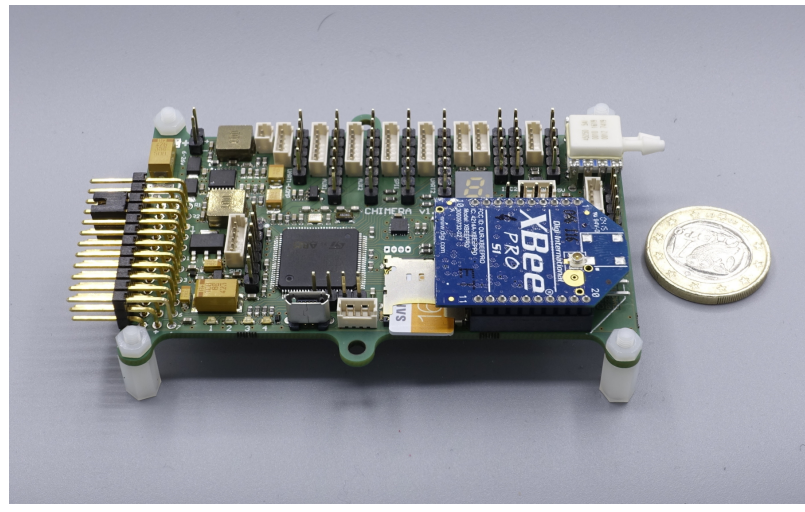

Figure 5. Overview of Chimera v1.0 autopilot board from Paparazzi Autopilot System

ARM Cortex M-7 CPU
9 DOF IMU (MPU 9250)
Barometer, Differential Pressure
High-Speed logging via SD card
$8 \times$ Servos (commercial plugs)
$5 \times$ UART
I2C , SPI, CAN
On-board XBee support
Companion Board ready
89 mm x $60 \mathrm{~mm}$
Table 2. General specifications of
Chimera v1.0

${ }^{a}$ wiki.paparazziuav.org/Chimera/v1.00 
The propulsion system total weight and efficiencies have been kept constant per motor during the conceptual design evaluations. An appropriate motor and propeller couple has been selected afterwards by using QPROP $^{14}$ and wind-tunnel experiments.

\section{B. Configuration}

The maximum dimension restrictions eliminate most of the possibilities for the configuration selection. As the main objective is high operational efficiency, the simplest and most compact configuration is a twinmotor plank wing. Most of the meteorological sensors requires a clean front end without propellers or other obstacles influencing the in coming flow. Therefore, a forward nose cone with sufficient inside volume for the payload placement is required. With these decisions made, the configuration is almost fixed, only the exact wing span and the surface area remain undefined. These quantities, as well as the battery weight, propeller dimensions, and control surface sizing are selected with the help of the explained methodology. Possible combinations of design variables are then evaluated using a computer program. Each candidate aircraft has to be able to satisfy steady flight equilibrium for the full flight speed envelope. Among all, the most compact vehicle dimensions have been selected.

\section{Airfoils}

During a typical mission, the aircraft flies with the propellers constantly blowing on the wing. Therefore the wing section within this propeller slipstream will be working in turbulent conditions. The transition from laminar to turbulent flow will also appear on the nose of the fuselage before the wing, which influences the portion of the wing around the fuselage joint. Adding the transition around the wingtip to these, it can be assumed that the complete wing will be always working in turbulent flow conditions.

Therefore the airfoils are designed for turbulent flow conditions by tripping the boundary layer artificially around the leading edge. The design process is done by using XFOIL interactively. The trigger point for the transition is selected to be at $0.05 \%$ of the chord length, both on the top and bottom surface of the wing. The starting point for the design was a combination of MH45 and S5010 airfoils. The trailing edge gap thickness is selected as $0.8 \mathrm{~mm}$ and set specifically according to the local chord of each airfoil. This value is mainly driven by the manufacturing technique used and the strength of the material on the trailing edge. The total thickness of the airfoil has the biggest influence on the performance, therefore the thickness for each section has been reduced as much as possible, without loosing too much on the spar strength. It has to be mentioned that as the spar gets thinner, the total weight increases for a given strength. The loading is modified on the aft part of the airfoil, so that an almost positive pitching moment is obtained for stable flight during cruise phase.

\section{Propulsion System}

After selecting the twin-motor configuration, the weight of each combination of motor, propeller, speed controller and required electrical cable is assumed to be approximately 90 grams during the iterations. This assumption relies on the previous experience with similar propulsion systems using same required power.

A good fit from the existing off-the-shelf propellers is found to be the $H Q$-Prop $8 \times 5$, and an off-the-shelf electric motor that has been used and tested in-house for several years is the AXI-2212/26. The Figure 6 shows the wind-tunnel test results of this motor/propeller combination at different speeds. The tests were stopped around $6 \mathrm{~N}$ of thrust, because of the limitations of the force sensor. The usage of a four-cell battery with $14.8 \mathrm{~V}$ results in approximately $10 \mathrm{~N}$ of thrust in static $(0 \mathrm{~m} / \mathrm{s})$ conditions, which will be sufficient for hovering capability, even if we take an additional control safety margin into account.

\section{E. Final Appearance}

Table 3 shows the final specifications, and Figure 7 shows the manufactured prototype of the Cyclone. It should be noted that, the first prototype is equipped with a different motor, which is AXI-2808/24 because of possible heating problems during extensive durations of hovering flight. In reality, the vehicle will not be hovering more than two minutes. 

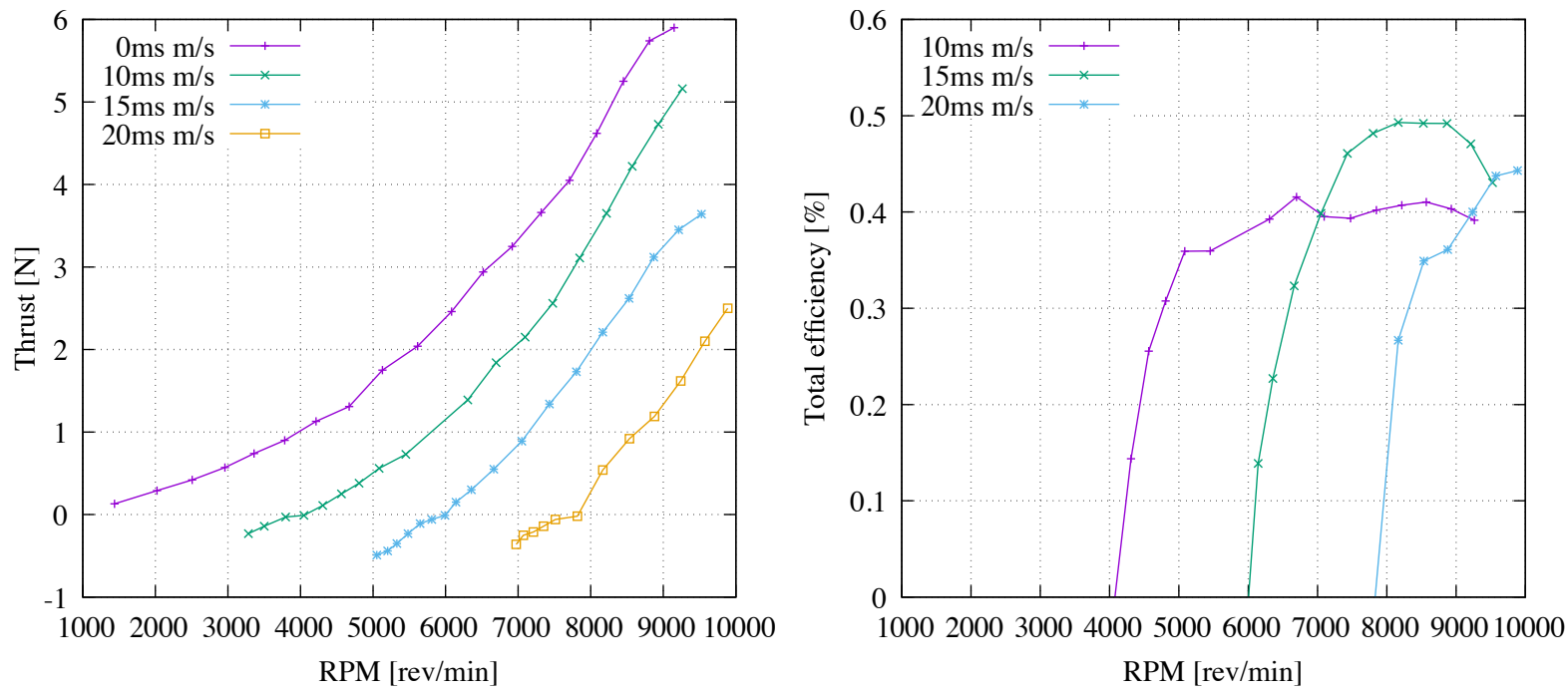

Figure 6. Thrust and total system efficiency versus RPM plots at different wind tunnel speeds for the AXI2212-26 electric motor and HQ-Prop $8 \times 5$ propeller combination.

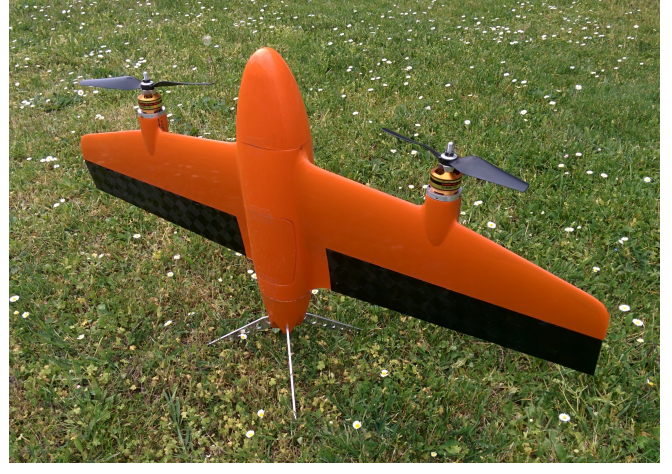

Figure 7. Overview of Cyclone

\begin{tabular}{lcl}
\hline Wing Span & 0.88 & {$[\mathrm{~m}]$} \\
Wing Surface Area & 0.15 & {$\left[\mathrm{~m}^{2}\right]$} \\
Mean Aero. Chord & 0.17 & {$[\mathrm{~m}]$} \\
Prop Diameter & 0.21 & {$[\mathrm{~m}]$} \\
Section Airfoil & custom & \\
Flight Speed Range & $0-30$ & {$[\mathrm{~m} / \mathrm{s}]$} \\
MTOW (hand-released) & 1.5 & {$[\mathrm{~kg}]$} \\
Payload Capacity & $0-0.4$ & {$[\mathrm{~kg}]$} \\
\hline
\end{tabular}

Table 3. General specifications of Cyclone 


\section{Manufacturing}

In order to achieve the requested high quality surface and precise geometry, the manufacturing is all done by high rpm CNC machined aluminum molds. This also helps to obtain the precision required by the thin turbulent airfoils. Composite materials are used in order to achieve a light weight and robust airframe. The skin of the wing is made up of Rohacell sandwich material covered with aramid on the top and glass fiber in the inside. The main spar is made from a carbon fiber sleeve, and the spar caps are layed up directly to the inner side of the wing skins, where the spar gets bonded afterwards. The inner reinforcements for the motor mounts and for the hinge location is shown in the most right picture of the Figure 8. Vacuum bag technique with wet lay-up is used, which is also shown in the third picture from the left in the Figure 8. Most of the electronics and the control servos are placed and fixed inside the aircraft during the closing of the top and bottom half of the molds together. The control surfaces are directly attached to the servo shafts on the root and aluminum housings and pins are used for the hinge mechanism. The nose cone and the tail cone are manufactured separately, and they can be removed for easy access to the internal payload and also for easy transportation.
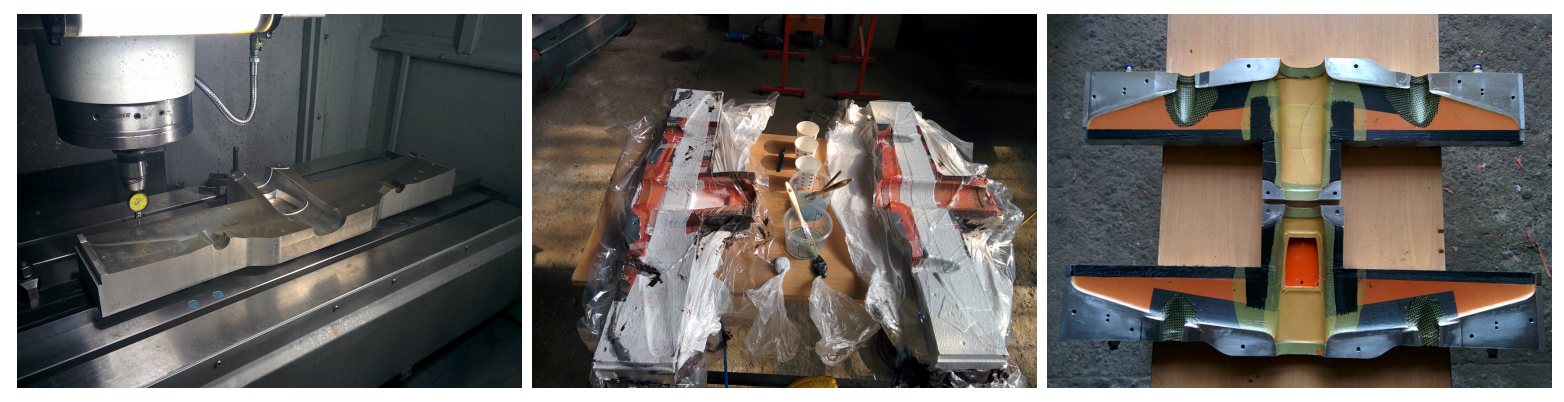

Figure 8. Manufacturing phases, CNC mold milling on the left, composite wet lay up vacuum bag in the middle, and finished skins on the right before closing the molds together.

\section{Control}

This section deals with the attitude control of the vehicle across the flight envelope. The axis definitions of the vehicle are based on the hover condition, as shown in Figure 9.

The Cyclone has four independent actuators that lead to four degrees of freedom for the control of the vehicle. The motors can provide a force in the negative body $\mathrm{Z}$ axis, as well as a moment around the body $\mathrm{X}$ axis. The flaps provide moments around the body $\mathrm{Y}$ and $\mathrm{Z}$ axes and can provide these moments even in hover flight, due to the propeller slipstream.

From test flight data it appears that the torque from the propellers is not very strong, possibly because of the interaction of the slipstream with the wing. Nonetheless, the propellers spin in opposite directions, such that the torques are canceled at equal rotational speed. Test flights show that mo-

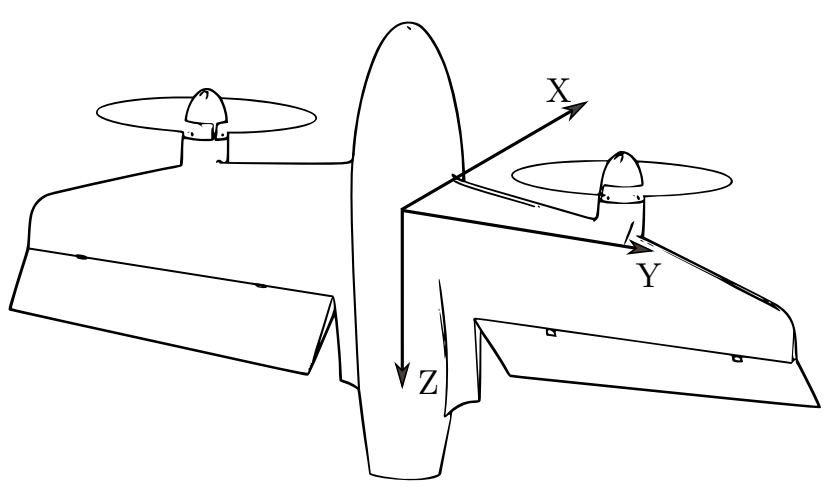

Figure 9. The body axis definitions of the Cyclone ments around the body $\mathrm{Z}$ axis due to typical changes in individual motor commands, caused by propeller drag and inertia, are relatively small.

To transition to and back from forward flight without changing altitude, the Cyclone encounters very large angles of attack, a stalled wing, and rapidly changing pitching moments. Modeling of the forces and moments at different airspeeds and different angles of attack is costly and time consuming. Furthermore, a controller based on this data will require accurate knowledge of the airspeed and angle of attack, which is difficult to obtain, especially in gusty conditions. That is why Incremental Nonlinear Dynamic Inversion (INDI) control method is chosen, which needs no modeling of the vehicle dynamics and is very strong at disturbance rejection. 


\section{A. Incremental Nonlinear Dynamic Inversion}

In this paper we present the first application of INDI to a transitioning UAV. The controller is based on the work of Smeur et al. ${ }^{15}$ and revolves around the control of the angular accelerations in an incremental way. We proceed with a brief overview of the chosen control technique, for more details we refer to mentioned paper.

First, consider the angular acceleration of the vehicle. Assuming that the gyroscopic moments can be neglected, the angular acceleration of the vehicle becomes a function of the state and the input vector $\boldsymbol{u}$ as follows

$$
\boldsymbol{I} \dot{\boldsymbol{\Omega}}=\boldsymbol{f}(\boldsymbol{\Omega}, \boldsymbol{v})+\boldsymbol{g}(\boldsymbol{u})
$$

where $\boldsymbol{I}$ is the moment of inertia matrix, which is assumed to be diagonal, $\boldsymbol{\Omega}$ is the angular rate vector and $\boldsymbol{v}$ is the airspeed vector. In order to invert this equation for the inputs $\boldsymbol{u}$ given a desired angular acceleration vector, the functions $\boldsymbol{f}$ and $\boldsymbol{g}$ need to be accurately known. So instead, a first order Taylor expansion can be applied to the (19) considering that the change in angular acceleration is caused by a change in the inputs and states, namely

$$
\boldsymbol{I} \dot{\boldsymbol{\Omega}}=\boldsymbol{I} \dot{\boldsymbol{\Omega}}_{0}+\left.\frac{\partial}{\partial \boldsymbol{\Omega}} \boldsymbol{f}\left(\boldsymbol{\Omega}, \boldsymbol{v}_{0}\right)\right|_{\boldsymbol{\Omega}=\boldsymbol{\Omega}_{0}}\left(\boldsymbol{\Omega}-\boldsymbol{\Omega}_{0}\right)+\left.\frac{\partial}{\partial \boldsymbol{v}} \boldsymbol{f}\left(\boldsymbol{\Omega}_{0}, \boldsymbol{v}\right)\right|_{\boldsymbol{v}=\boldsymbol{v}_{0}}\left(\boldsymbol{v}-\boldsymbol{v}_{0}\right)+\left.\frac{\partial}{\partial \boldsymbol{u}} \boldsymbol{g}(\boldsymbol{u})\right|_{\boldsymbol{u}=\boldsymbol{u}_{0}}\left(\boldsymbol{u}-\boldsymbol{u}_{0}\right)
$$

This equation describes how to predict the angular acceleration knowing the current angular acceleration, the partial derivatives of the functions $\boldsymbol{f}$ and $\boldsymbol{g}$ and the change in state and inputs. Here, the current angular acceleration is caused by the sum of moments from inputs, state and disturbances. Assume that we are looking only a small time ahead, but long enough for the actuators to adopt their new values. We assume that in this small time period, the change in $\boldsymbol{\Omega}$ and $\boldsymbol{v}$ times their respective derivatives can be neglected. If we divide by $\boldsymbol{I}$ and assume that the partial derivative of $\boldsymbol{g}$ with respect to $\boldsymbol{u}$ can be approximated by a static matrix for some part of the flight envelope, we arrive at

$$
\dot{\boldsymbol{\Omega}}=\dot{\boldsymbol{\Omega}}_{0}+\boldsymbol{G}\left(\boldsymbol{u}-\boldsymbol{u}_{0}\right)
$$

where $\boldsymbol{G}$ is the control effectiveness matrix, which contains the effectiveness of each actuator on each axis. Its values are determined with a least squares fit with changes in input and angular acceleration obtained from flight data. The control law can now be obtained by inverting (21):

$$
\boldsymbol{u}=\boldsymbol{u}_{0}+\boldsymbol{G}^{-1}\left(\boldsymbol{\nu}-\dot{\boldsymbol{\Omega}}_{0}\right)
$$

where the desired angular acceleration has become the virtual control $\boldsymbol{\nu}$. Since the angular acceleration is now controlled, we can design a PD controller that will provide the virtual control, in order to control the attitude. We have shown that the gains of this PD controller, if the assumptions hold, depend only on the actuator dynamics. ${ }^{16}$

Of course the effectiveness of the actuators varies over the flight envelope. Especially the aerodynamic surfaces become much more effective with increasing airspeed. To cope with this, we are changing the control effectiveness of the flaps according to a predefined function of the square of the airspeed. This does require measurement of the airspeed throughout the flight envelope. For this purpose, a Pitot tube is mounted on the nose of the aircraft, pointing in the negative body $\mathrm{Z}$ axis. This sensor can not deliver accurate measurements at low speeds and high angle of attack. Therefore, if the airspeed sensor measures less than six $\mathrm{m} / \mathrm{s}$, we use the pitch angle to calculate the flap effectiveness.

Angle of attack and sideslip vanes are also mounted on the nose of the aircraft, though they are not used for control. The data from these sensors is just used to evaluate the flights.

\section{B. Modeling of the Actuators}

Although with INDI we do not model increments in external moments, it is important to model the control inputs. Consequently, a model of the actuator dynamics is essential. This will tell the controller what the expected response is for the input increments he applies. The Cyclone is equipped with DS6125MINI servos, directly attached to the control surfaces. To determine the response of these servos, we attached a wire to what was believed to be the internal potentiometer signal, and read out the voltage using a logic analyzer. For some step inputs at no load, the output signal could be roughly modeled with

$$
A(z)=\frac{\alpha}{z-(1-\alpha)}
$$


where $\alpha=0.1$ for a sample frequency of $512 \mathrm{~Hz}$ and a rate limit of 272 degrees per second. We also measured a delay of ten samples, but adding this in the model gave a slightly worse fit with the angular acceleration measured in test flights. Therefore, the delay is not included in the final actuator model, and more investigation is needed to determine whether it should be taken into account or not.

For the motors that drive the propellers, the actuator model is estimated just by fitting the angular acceleration in roll with the signal to the motors filtered by equation (23), without a rate limit. The best fit was obtained with $\alpha=0.05$. However, the tracking of angular accelerations is a bit slower than can be expected based on this actuator model, so possibly this value of $\alpha$ is not very accurate. In practice, this means that the $\mathrm{P}$ and $\mathrm{D}$ gains, that are normally designed based on the actuator model, need to be less aggressive to avoid oscillations.

\section{Generating Pitching Moment}

One of the main difficulties of the design is to provide enough pitch-up moment to counteract the natural pitch-down moment of a flying wing, specifically at high angle of attack flight. Even if an equilibrium can be found for every velocity and angle of attack, disturbances or variations in the angle of attack and airspeed will require some additional control margin. This is a particular problem for the type of vehicle presented in this paper, as other vehicles equipped with multiple rotors or a swash plate can typically provide stronger moments.

The center of gravity is placed close to the neutral point of the vehicle. Therefore, a potential solution could be to move it more to the back. However, this results in a vehicle that is very unstable in forward flight, dramatically reducing the control performance in this flight phase.

Another potential solution would be to create a pitch-up moment with the propellers, by changing the value of $d_{T}$ (see Figure 4). Without any servo mechanism, this moment would be static. This means that it may help when the vehicle pitches forward, but it will make things worse when pitching backward.

\section{Descending while Hovering}

Since the flaps rely on the slipstream of the propellers for dynamic pressure in hover flight, a large reduction in thrust will lead to a loss of flap effectiveness. While hovering, achieving a stable vertical descent is quite delicate. This is why the pilot, or the autopilot, should never give very low throttle. Additionally, a rapid vertical descent can lead to flow reversal over the wing, leading to inversion of the flap effectiveness. This will lead to the vehicle yawing and pitching the wrong way.

The pilot, or autopilot, can bring the vehicle back to a stable condition simply by adding thrust. With higher thrust, the propeller slipstream will increase, leading to an increase in dynamic pressure on the flaps. Additionally, the added thrust will result in an increase in airspeed, higher dynamic pressure and therefore an increase in the flap effectiveness.

\section{Flight Tests}

In order to demonstrate the viability of the vehicle, together with the proposed incremental control method, and to show that the vehicle is able to hover, fly forward and gradually transition between the two, we have conducted a series of flight tests. The preliminary flight tests were performed with a development version of the Cyclone made out of foam, aramid and 3D printed pieces. A picture of the development version is shown in Figure 10.

Figure 11 shows several variables during a flight in which the ground speed was commanded by a pilot. The first two graphs show the desired and measured pitch and roll angles respectively. Note that the rotation order of the Euler angles used is 'ZXY', instead of the more conventional 'ZYX', in order to avoid the singularity at ninety degrees pitch angle. ${ }^{17}$ The vehicle starts out hovering with a pitch angle of around -33 degrees, because of the presence of a bit of wind. Then an increasing velocity is commanded, and the aircraft accelerates to $23 \mathrm{~m} / \mathrm{s}$ airspeed. While in forward flight, several turns are performed, as can be seen from the roll angle.

We also show the deflection of both flaps in the third graph of Figure 11. A positive deflection yields a pitch-up moment and the maximum deflection is 30 degrees. Note how the vehicle needs considerable flap deflections in order to avoid pitching down at low speed and pitch angles between -20 and -70 degrees. Especially when additional pitch up moment is required, this can lead to saturations, as can be seen in the 

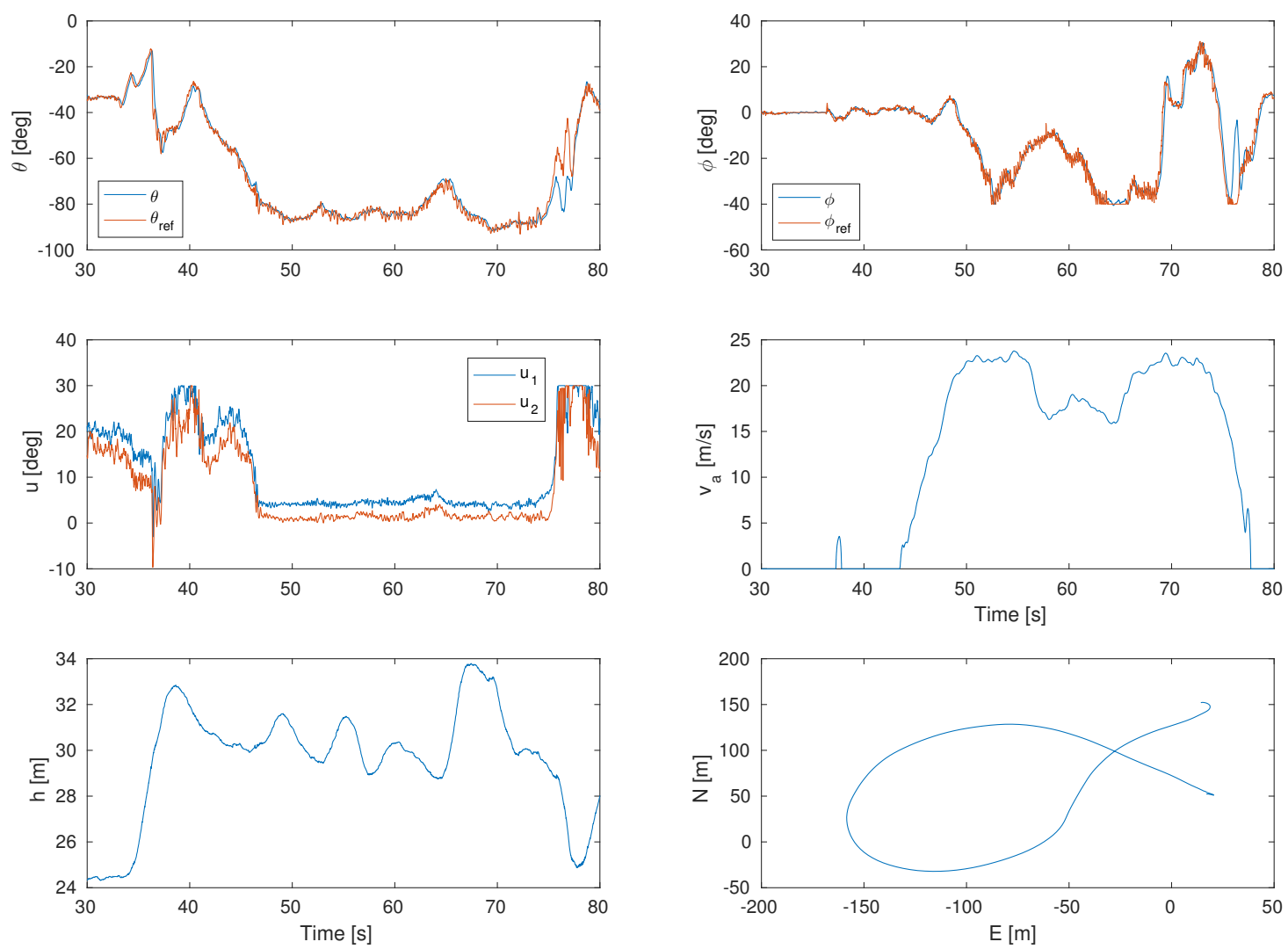

Figure 11. Flight test of the Cyclone. On the left side, from top to bottom: pitch angle, flap deflection and the height above ground. On the right side: roll angle, measured airspeed and a top view of the trajectory.

figure at 40 seconds and at the end of the forward flight phase at 75 seconds. The tracking of the pitch and roll reference is good throughout the flight, except when transitioning back from forward flight.

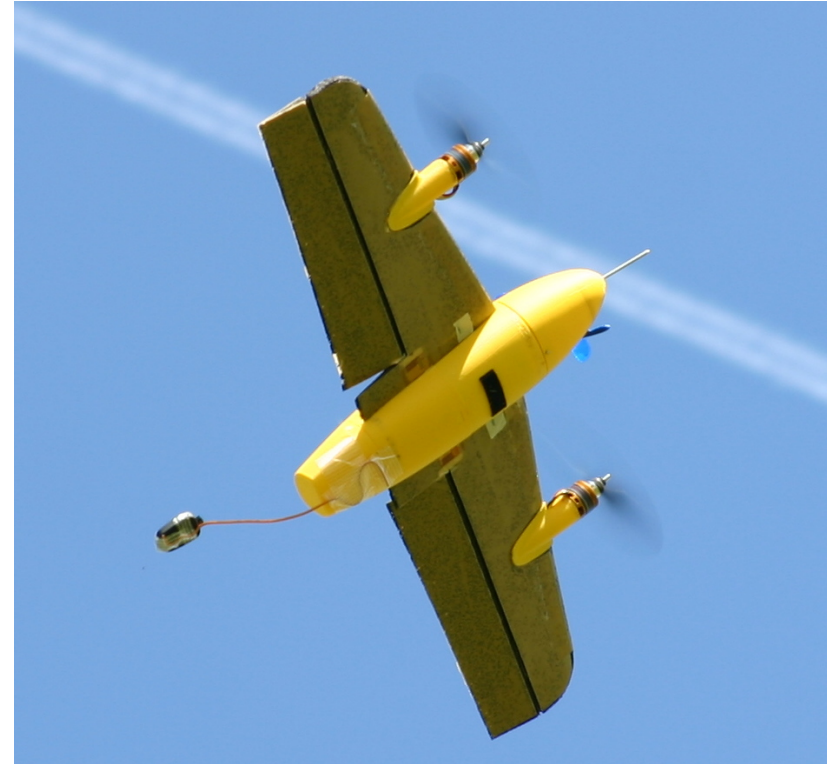

Figure 10. The development version of the Cyclone, in flight with a camera mounted on the rear.
In this part of the flight, the vehicle needs to pitch up more than the flaps can physically achieve. We prioritize pitch over roll, which is why upon saturation the roll control suffers as well. Additionally, we change the effectiveness of the propellers on the pitch axis to a nonzero value when both flaps are almost saturated. This makes the vehicle add thrust in order to pitch up, which increases the propeller slipstream velocity, making the flaps more effective. Also, at high angle of attack, increasing the thrust typically reduces the angle of attack, which reduces the aerodynamic pitch moment.

These problems can be reduced by increasing the altitude during the transition, such that the angle of attack and the aerodynamic pitch moment remain small. However, we require the vehicle to be able to cope with strong winds, which requires flying at a large angle of attack as well. In this case, the vehicle is even descending when transitioning back to hover.

Finally, we highlight the satisfactory tracking of pitch and roll during the first part of the flight with the (non-saturated) required flap deflections. We recall that we have not modeled the aerodynamic moments and still the vehicle is able to counteract them, 
even when the maximum flap deflections are required after 40 seconds of flight. This experiment shows that the INDI control technique is able to cope with the unmodeled moments, even though they are strong compared to the control input.

Although the guidance and navigation is not the main topic of this paper, Figure 12 shows preliminary results of the capability of the Cyclone to autonomously travel over four waypoints in a square at $20 \mathrm{~m} / \mathrm{s}$. The UAV is programmed to aim its ground speed vector to the current waypoint, and it switches to the next waypoint (counter clockwise) when it has a distance of $30 \mathrm{~m}$ to the current waypoint. There was a mild wind of approximately $4 \mathrm{~m} / \mathrm{s}$ coming from West, which explains the asymmetry in the turns.

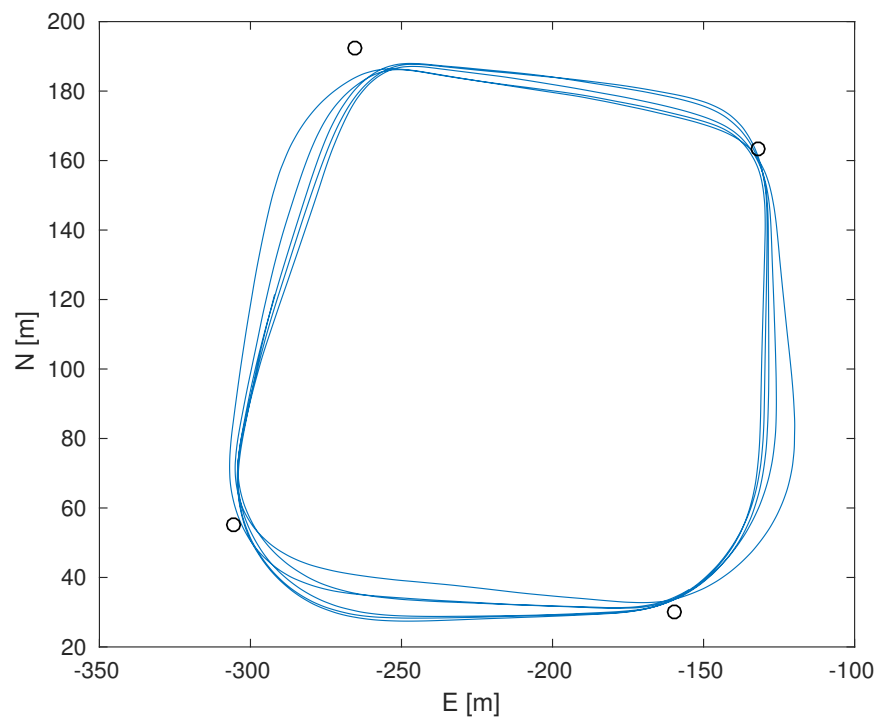

Figure 12. Ground track of navigation in forward flight at $20 \mathrm{~m} / \mathrm{s}$.

\section{Conclusion and Future Work}

Within the objective of optimizing a fixed wing mini-UAV with transitioning flight capability, a brief description of the semi-empirical method for estimating force and moments generated by a wing, partially or fully immersed inside distributed propeller slipstream is presented. The method is written as a program and used during the conceptual design phase for performance evaluations of blown-wing type configuration. According to the given mission requirements, an $88 \mathrm{~cm}$ wing span vehicle, called Cyclone, is designed.

The applicability of incremental nonlinear dynamic inversion control to a hybrid vehicle is shown. This control method does not depend on an extensive model of the vehicle, which makes it very easy to apply to such a complex vehicle. Only a model of the actuators and the control effectiveness are needed, which are relatively easy to obtain. Obtaining a model of the moments across the flight envelope through CFD or windtunnel tests can be very expensive and time consuming, so avoiding this is beneficial.

Test flights are employed to evaluate the controller implementation and show the viability of the aircraft design. The experiments show the ability of the Cyclone to hover, transition and fly forward, with good attitude tracking. However, poor capability of generating positive pitching moment using aerodynamic control surfaces has also been highlighted. This can lead to problems when descending with high angle of attack, which may be necessary on windy conditions.

Therefore, future work will include improving the pitch moment generation without losing efficiency and an in-depth analysis of the control of the attitude and position using INDI. Finally, the designed 90 minutes of endurance capability has to be proven with additional flight tests.

\section{Acknowledgments}

Development of the prototype vehicle has been partly funded by the MISTRALE Project. The authors would like to thank Xavier Paris for his contribution on the experimental setup and flight tests. 


\section{References}

${ }^{1}$ Jensen, A. M., Hardy, T., McKee, M., and Chen, Y., "Using a multispectral autonomous unmanned aerial remote sensing platform (AggieAir) for riparian and wetlands applications," IEEE International Geoscience and Remote Sensing Symposium, July 2011.

${ }^{2}$ Bronz, M., Hattenberger, G., and Moschetta, J.-M., "Development of a Long Endurance Mini-UAV: ETERNITY," International Journal of Micro Air Vehicles, Vol. 5, No. 4, 2013, pp. 261-272.

${ }^{3}$ Bronz, M. and Hattenberger, G., "Design of A High-Performance Tailless MAV Through Planform Optimization," 33rd AIAA Applied Aerodynamics Conference, AIAA, 2015, pp. eISBN-978.

${ }^{4}$ Lustosa, L., Defay, F., and Moschetta, J.-M., "Development of the flight model of a tilt-body MAV," 2014 International Micro Air Vehicle Conference and Competition (IMAV 2014), Delft, NL, 2014, pp. 157-163, Thanks to IMAV 2014. The original PDF is available at TU Delft Library Open Access repository: http://repository.tudelft.nl/conferencepapers/.

${ }^{5}$ Hartmann, P., Schtt, M., and Moormann, D., "Control of Departure and Approach Maneuvers of Tiltwing VTOL Aircraft," Guidance, Navigation, and Control Conference, AIAA 2017-1914, 2017.

${ }^{6}$ De Wagter, C., Ruijsink, R., Smeur, E., van Hecke, K., van Tienen, F., van der Horst, E., and Remes, B., "Design, Control and Visual Navigation of the DelftaCopter," arXiv:1701.00860v1, January 2017.

${ }^{7}$ Gregory, I. M., Ackerman, K., Snyder, S., and Rothhaar, P., "Adaptive control for tilt-wing VTOL UAV," 2015 American Control Conference (ACC), July 2015, pp. 2535-2535.

${ }^{8}$ Veldhuis, L. L. M., Propeller wing aerodynamic interference, TU Delft, Delft University of Technology, 2005.

${ }^{9}$ Jameson, A., "The Analysis of Propeller Wing Flow Interaction, Analytic Methods in Aircraft Aerodynamics," NASA Symposium Proceedings SP-228, October 1969, pp. 721-749.

${ }^{10}$ Bronz, M. and Drouin, A., "Preliminary Design Estimation of the V/STOL Airplane Performance," IMAV 2015, International Micro Air Vehicles Conference and Flight Competition, Aachen, September 2015.

${ }^{11}$ Ribner, H. S., "Notes on the Propeller Slipstream in Relation to Stability," NACA ARR L4112a (WRL-25), 1944.

${ }^{12}$ De Young, J., "Propellers at High Incidence," Journal of Aircraft, Vol. 2, No. 3, May-June 1965, pp. pp 241-250.

${ }^{13}$ Draper, J. W. and Kuhn, R. E., "Investigation of the Aerodynamic Characteristics of a model wing-propeller combination and of the wing and Propeller Separately at Angles of Attack up to 90 degrees," Tech. Rep. NACA-TN-3304, National Advisory Committee for Aeronautics. Langley Aeronautical Lab., Langley Field, VA, United States, November 1954, ID:19930092264.

${ }^{14}$ Drela, M., QPROP Formulation, MIT Aero and Astro, June 2006.

${ }^{15}$ Smeur, E. J. J., Chu, Q. P., and de Croon, G. C. H. E., "Adaptive Incremental Nonlinear Dynamic Inversion for Attitude Control of Micro Aerial Vehicles," Journal of Guidance, Control, and Dynamics, Vol. 39, No. 3, March 2016, pp. 450-461.

${ }^{16}$ Smeur, E. J. J., de Croon, G. C. H. E., and Chu, Q. P., "Cascaded Incremental Nonlinear Dynamic Inversion Control for MAV Disturbance Rejection," January 2017.

${ }^{17}$ Oosedo, A., Abiko, S., Konno, A., and Uchiyama, M., "Optimal transition from hovering to level-flight of a quadrotor tail-sitter UAV," Autonomous Robots, Vol. 41, No. 5, 2017, pp. 1143-1159. 